\title{
Comparing tamed and compatible symplectic cones and cohomological properties of almost complex manifolds
}

\author{
Tian-Jun Li AND WeIYi Zhang
}

\begin{abstract}
We introduce certain homology and cohomology subgroups for any almost complex structure and study their pureness, fullness and duality properties. Motivated by a question of Donaldson, we use these groups to relate $J$-tamed symplectic cones and $J$ compatible symplectic cones over a large class of almost complex manifolds, including all Kähler manifolds, almost Kähler 4manifolds and complex surfaces.
\end{abstract}

1 Introduction

2 Some homology and cohomology subgroups of almost complex manifolds

2.1 Forms and currents

2.2 Pureness and fullness

2.2.1 $C^{\infty}$ pure and full almost complex structures

2.2.2 Pure and full almost complex structures

2.2.3 Closed almost complex structures

\subsection{Duality}

3 Symplectic forms versus complex cycles

3.1 Complex cycles and tamed symplectic forms 


\subsubsection{Complex cycles}

3.1.3 Tamed symplectic forms

3.2 Complex cycles and compatible symplectic forms

3.3 Comparing $\mathcal{K}_{J}^{c}$ and $\mathcal{K}_{J}^{t}$

4 Complex structures

4.1 Kähler complex structures

4.1.1 Tamed cones and compatible cones

4.1.2 Relating to some classical theorems

4.2 Complex surfaces

4.2.1 Tamed cones and compatible cones

4.2.2 Integrable tamed cone versus integrable compatible cone

References

680

\section{Introduction}

Let $M$ be a closed oriented smooth $2 n$-manifold. A symplectic form compatible with the orientation is a closed 2 -form $\omega$ such that $\omega^{n}$ is a volume form compatible with the given orientation. Let $\Omega_{M}$ be the space of such 2-forms. By taking the cohomology class, we have the projection map cс $: \Omega_{M} \longrightarrow H^{2}(M ; \mathbb{R})$. The image $\operatorname{cc}\left(\Omega_{M}\right) \subset H^{2}(M)$ is called the symplectic cone of $M$, and is denoted by $\mathcal{C}_{M}$. In recent years, $\mathcal{C}_{M}$ has been extensively studied, especially in dimension 4. In [23], McDuff determined $\mathcal{C}_{M}$ for all the ruled surfaces. A complete description for the symplectic cone of all the 4 -manifolds with $b^{+}=1$ was subsequently given in [21]. After that, several attempts in different directions were made, e.g., [22] and [8].

In this note, instead of only fixing an orientation, we further fix a specific almost complex structure $J$ compatible with the given orientation. That is to 
say, we consider an almost complex $2 n$-manifold $(M, J)$. Correspondingly, we study the following subcones of $\mathcal{C}_{M}$ associated to the almost complex structure $J$.

Definition 1.1. The $J$-tamed symplectic cone is

$$
\mathcal{K}_{J}^{t}=\left\{[\omega] \in H^{2}(M ; \mathbb{R}) \mid \omega \text { is tamed by } J\right\},
$$

and the $J$-compatible symplectic cone is

$$
\mathcal{K}_{J}^{c}=\left\{[\omega] \in H^{2}(M ; \mathbb{R}) \mid \omega \text { is compatible with } J\right\}
$$

Recall that $J$ is an automorphism of the tangent bundle $T M$ satisfying $J^{2}=$-id, and $J$ is said to tame $\omega$ if $\omega$ is positive on any $J$-line $\operatorname{span}(v, J v)$ where $v$ is a non-zero tangent vector. An $\omega$-tamed $J$ is said to be compatible with $J$ if, further, $\omega$ is $J$-invariant. Since $J$-tameness is an open condition and is preserved under convex combinations, $\mathcal{K}_{J}^{t}$ is an open convex cone in $H^{2}(M ; \mathbb{R})$. Moreover, cohomologous $J$-tamed forms are isotopic. Since $J$ compatibility is also preserved under convex combinations, $\mathcal{K}_{J}^{c}$ is a convex subcone of $\mathcal{K}_{J}^{t}$.

There is a notion of integrability of $J$ given by the vanishing of the Nijenhuis tensor. The deep Newlander-Nirenberg Theorem asserts that $J$ is integrable if and only it is induced by a complex structure. For a complex structure $J$, there is the Dolbeault complex and the associated Dolbeault groups $H_{\bar{\partial}}^{p, q}(M)$. The $J$-compatible cone $\mathcal{K}_{J}^{c}$ for a complex structure is just the usual Kähler cone. We call a complex structure $J$ Kähler if its Kähler cone is non-empty. In this case, the Kähler cone is an open convex cone of $H_{\bar{\partial}}^{1,1}(M)_{\mathbb{R}}$, where

$$
H_{\bar{\partial}}^{p, q}(M)_{\mathbb{R}}=H_{\bar{\partial}}^{p, q}(M) \cap H^{p+q}(M ; \mathbb{R}) .
$$

More generally, we call $J$ almost Kähler if its compatible cone is not empty. Almost Kähler manifolds have been studied from the point of view of Riemannian geometry ever since Gray. The emphasis is often on a fixed compatible metric $g$ and its curvature properties, see, e.g., [1]. Our point of view is different as we do not fix a priori a metric.

Part of our motivation for studying these cones is due to a question raised by Donaldson in [7].

Question 1.1. If $J$ is an almost complex structure on a compact 4-manifold which is tamed by a symplectic form, is there a symplectic form compatible with $J$ (and in the same cohomology class if $b^{+}=1$ )? 
Deep results on this question have been made in [32] and [30]. In our language this question is, if $\mathcal{K}_{J}^{t}$ is not-empty for some $J$, then is $\mathcal{K}_{J}^{c}$ nonempty as well? This question only makes sense in dimension 4 , because, for $n \geq 3$, $\mathcal{K}_{J}^{c}$ is empty even locally for a generic almost complex structure (cf. [19] and $[31])$.

Also motivated by Question 1.1, we introduce in Section 2 the analogues of the (real) Dolbeault groups

$$
H_{\bar{\partial}}^{1,1}(M)_{\mathbb{R}} \quad \text { and } \quad\left(H_{\bar{\partial}}^{2,0}(M) \oplus H_{\bar{\partial}}^{0,2}(M)\right)_{\mathbb{R}}
$$

for general $J$, which we denote by

$$
H_{J}^{1,1}(M)_{\mathbb{R}} \quad \text { and } \quad H_{J}^{(2,0),(0,2)}(M)_{\mathbb{R}}
$$

respectively (see Definition 2.4).

An important observation related to Question 1.1 is, as in the integrable case, the $J$-compatible cone is an open (possibly empty) convex cone of $H_{J}^{1,1}(M)_{\mathbb{R}}$.

We analyze for what $J$ the groups in (1.1) give rise to a direct sum decomposition of $H^{2}(M ; \mathbb{R})$. Such a $J$ is called $C^{\infty}$ pure and full. ${ }^{1}$

We also introduce the corresponding groups for currents

$$
H_{1,1}^{J}(M)_{\mathbb{R}} \quad \text { and } \quad H_{(2,0),(0,2)}^{J}(M)_{\mathbb{R}}
$$

and introduce the notion of pure and full when the groups in (1.2) give rise to a direct sum decomposition of $\mathrm{H}_{2}(M ; \mathbb{R})$.

Using ideas in [28] and [16] we prove in Section 3

Theorem 1.1. Suppose $J$ is a $C^{\infty}$ full almost complex structure, which means that $H_{J}^{1,1}(M)_{\mathbb{R}}$ and $H_{J}^{(2,0),(0,2)}(M)_{\mathbb{R}}$ generate $H^{2}(M ; \mathbb{R})$. If $\mathcal{K}_{J}^{c}$ is nonempty then

$$
\mathcal{K}_{J}^{t}=\mathcal{K}_{J}^{c}+H_{J}^{(2,0),(0,2)}(M)_{\mathbb{R}}
$$

We want to point out that Theorem 1.1 applies to Kähler complex structures. And in this case, it is not hard to see that $H_{J}^{(2,0),(0,2)}(M)_{\mathbb{R}}$ is isomorphic to $\left(H_{\bar{\partial}}^{2,0}(M) \oplus H_{\bar{\partial}}^{0,2}(M)\right)_{\mathbb{R}}[11]$.

For general almost complex structures the cohomology subgroups in (1.1) and their homology analogues in (1.2) seem to have not been systematically

\footnotetext{
${ }^{1}$ The use of $C^{\infty}$ here (as well as in Definitions 2.2, 2.3 and 2.6) indicates that we are dealing with forms rather than currents. It has nothing to do with the $C^{\infty}$ smoothness of $J$.
} 
explored in the literature. We believe that they are important invariants of almost complex structures and deserve further study. We would like to mention that there are two recent papers $[11,12]$ that are closely related to this work (see Remark 2.3). In particular, it is shown in [11] that any 4-dimensional almost complex structure is $C^{\infty}$ pure and full. It is easy to observe that $H_{J}^{(2,0),(0,2)}(M)_{\mathbb{R}}$ is always trivial if $b^{+}(M)=1$. Together with Theorem 1.1, we obtain,

Corollary 1.1. Suppose $(M, J)$ is an almost complex 4-manifold with nonempty $\mathcal{K}_{J}^{c}$. Then

$$
\mathcal{K}_{J}^{t}=\mathcal{K}_{J}^{c}+H_{J}^{(2,0),(0,2)}(M)_{\mathbb{R}}
$$

In particular, if $b^{+}(M)=1$, then $K_{J}^{t}=K_{J}^{c}$.

This provides some positive evidence for Question 1.1, especially in the case of $b^{+}=1$.

When $b^{+}>1$, the calculations in [11] also demonstrate that $H_{J}^{(2,0),(0,2)}$ $(M)_{\mathbb{R}}$ often vanishes. ${ }^{2}$ Thus, in light of Corollary 1.4 , we speculate that we might actually have the equality $\mathcal{K}_{J}^{t}=\mathcal{K}_{J}^{c}$ for a generic 4 -dimensional almost complex structure.

In Section 4 we focus on complex structures. We observe in this case Theorem 1.1 is also a direct consequence of the deep Nakai-Moishezon type Kähler criterion of [5]. ${ }^{3}$ We also point out the parallel to some classical results in algebraic geometry and Kähler geometry. Further, if we let $H \mathfrak{C}(J)$ be the cone of homology complex cycles, in the sense of Sullivan [28], then we can describe it using the $J$-compatible symplectic cone and the analytic subsets of $M$.

Moreover, for complex surfaces, we confirm Question 1.1.

Theorem 1.2. Let $J$ be a complex structure on a 4-manifold $M$. Then $\mathcal{K}_{J}^{t}(M)$ is empty if and only if $\mathcal{K}_{J}^{c}(M)$ is empty.

It is a direct consequence of several remarkable results in complex surface theory: the Kodaira classification [4], the Kähler criterion of $b^{+}$being

\footnotetext{
${ }^{2}$ It is also shown in [11] that $H_{J}^{2,0}+H_{J}^{0,2}=0$ whenever $J$ is not integrable. However, the group $\left(H_{J}^{2,0} \oplus H_{J}^{0,2}\right) \cap H^{2}(M ; \mathbb{R})$ is actually different from $H_{J}^{(2,0),(0,2)}(M)_{\mathbb{R}}$, and there are cases where the former group vanishes but the latter is non-trivial.

${ }^{3}$ And $[3,17]$ when $n=2$.
} 
odd $[3,24,27,29]$, and the analysis of complex curves in non-Kähler elliptic surfaces [16].

Finally we compare the union of tamed cones and the union of compatible cones over all complex structures in dimension 4 .

\section{Some homology and cohomology subgroups of almost complex manifolds}

We study in this section decompositions of forms and currents on almost complex manifolds and the associated cohomology and homology subgroups.

\subsection{Forms and currents}

On a smooth closed manifold $M$, the space $\Omega^{*}(M)$ of $C^{\infty}$ form is a vector space, and with $C^{\infty}$ topology, it is a Fréchet space, i.e., a complete metrizable locally convex topological vector space. The space $\mathcal{E}_{*}(M)$ of currents is the topological dual space, which is also a Fréchet space. As a topological vector space, $\Omega^{*}(M)$ is reflexive, thus it is also the dual space of $\mathcal{E}_{*}(M)$.

The exterior derivative on $\Omega^{*}(M)$ induces a boundary operator on $\mathcal{E}_{*}(M)$, making it also into a complex. By abusing notation we denote both the differentials in the current complex and the form complex by $d$.

Denote the space of closed forms by $\mathbf{Z}$ and the space of exact forms by B. Denote the space of closed currents by $\mathcal{Z}$ and the space of boundaries by $\mathcal{B}$. $\mathbf{Z}$ and $\mathcal{Z}$ are closed subspaces since $d$ is continuous. It is easy to check a current is closed if and only if it vanishes on $\mathbf{B}$, and a form is closed if and only if it vanishes on space $\mathcal{B}$.

We call the homology groups of the complex of currents the De Rham homology groups. The inclusion of smooth forms into the currents induces a natural isomorphism of the $(2 n-k)$-th De Rham cohomology group and the $k$-th De Rham homology group. Thus each closed $k$-current is homologous to a smooth $(2 n-k)$-form. Moreover, by Theorem $17^{\prime}$ in [6], a current is a boundary if and only if it vanishes on $\mathbf{Z}$, and a smooth form is a boundary if and only if it vanishes on $\mathcal{Z}$ (see [6]). This implies in particular that both $\mathbf{B}$ and $\mathcal{B}$ are also closed subspaces.

Let $J$ be an almost complex structure on a smooth manifold $M$. This means that $J$ is an automorphism of the tangent bundle $T M$ satisfying $J^{2}=-$ id. 
Extend the automorphism complex linearly to the complexified tangent bundle $T M_{\mathbb{C}}=T M \otimes \mathbb{C}$, we get a decomposition of $T M_{\mathbb{C}}$ into $\pm \mathbf{i}$ eigenbundles. In turn, we get a dual decomposition of the complexified cotangent bundle $T M_{\mathbb{C}}^{*}$. Denote the space of complex smooth differential $k$-forms $\Omega^{k}(M) \otimes \mathbb{C}$ by $\Omega^{k}(M)_{\mathbb{C}}$. The aforementioned decomposition of $T M_{\mathbb{C}}^{*}$ gives rise to a topological decomposition $\Omega^{1}(M)_{\mathbb{C}}=\Omega_{J}^{1,0}(M)_{\mathbb{C}} \oplus \Omega_{J}^{0,1}(M)_{\mathbb{C}}$. In turn, for each non-negative integer $k$, we have a topological type decomposition

$$
\Omega^{k}(M)_{\mathbb{C}}=\oplus_{p+q=k} \Omega_{J}^{p, q}(M)_{\mathbb{C}} .
$$

On the other hand, if $k$ is even, $J$ also acts naturally on $\Omega^{k}(M)$ as an involution and decomposes $\Omega^{k}(M)$ into the topological direct sum of the invariant part $\Omega_{J}^{k,+}(M)$ and the anti-invariant part $\Omega_{J}^{k,-}(M)$ with respect to this action. This action is given in the following way: for any $k$-form $\alpha$, and tangent vectors $v_{i}, i=1, \ldots, k$,

$$
(J \alpha)\left(v_{1}, \ldots, v_{k}\right)=\alpha\left(J v_{1}, \ldots, J v_{k}\right)
$$

We are particularly interested in the case $k=2$. In this case, the two decompositions are related in the following way:

$$
\begin{aligned}
& \Omega_{J}^{2,+}(M)=\Omega_{J}^{1,1}(M)_{\mathbb{R}} \quad:=\Omega_{J}^{1,1}(M)_{\mathbb{C}} \cap \Omega^{2}(M), \\
& \Omega_{J}^{2,-}(M)=\Omega_{J}^{(2,0),(0,2)}(M)_{\mathbb{R}}:=\left(\Omega_{J}^{2,0}(M)_{\mathbb{C}} \oplus \Omega_{J}^{0,2}(M)_{\mathbb{C}}\right) \cap \Omega^{2}(M) \text {. }
\end{aligned}
$$

Let $\mathbf{Z}_{J}^{1,1} \subset \Omega_{J}^{1,1}(M)_{\mathbb{R}}$ be the subspace of real closed $(1,1)$ forms, and $\mathbf{B}_{J}^{1,1} \subset$ $\Omega_{J}^{1,1}(M)$ be the subspace of real exact $(1,1)$ forms. Similarly define subspaces of $\Omega_{J}^{(2,0),(0,2)}(M)_{\mathbb{R}}, \mathbf{Z}_{J}^{(2,0),(0,2)}$ and $\mathbf{B}_{J}^{(2,0),(0,2)}$.

For the space of real 2-currents, we have a similar decomposition,

$$
\mathcal{E}_{2}(M)=\mathcal{E}_{1,1}^{J}(M)_{\mathbb{R}} \oplus \mathcal{E}_{(2,0),(0,2)}^{J}(M)_{\mathbb{R}},
$$

and the corresponding subspaces of closed, and boundary currents,

$$
\begin{array}{lllll}
\mathcal{B}_{1,1}^{J} & \subset & \mathcal{Z}_{1,1}^{J} & \subset & \mathcal{E}_{1,1}^{J}(M)_{\mathbb{R}} \\
\mathcal{B}_{(2,0),(0,2)}^{J} & \subset & \mathcal{Z}_{(2,0),(0,2)}^{J} & \subset & \mathcal{E}_{(2,0),(0,2)}^{J}(M)_{\mathbb{R}}
\end{array}
$$

We note the dual space of $\mathcal{E}_{1,1}^{J}(M)_{\mathbb{R}}$ is $\Omega_{J}^{1,1}(M)_{\mathbb{R}}$, and vice versa. The same can be said for $\mathcal{E}_{(2,0),(0,2)}^{J}(M)_{\mathbb{R}}$ is $\Omega_{J}^{(2,0),(0,2)}(M)_{\mathbb{R}}$. Since closed subspaces of a Fréchet space is Fréchet, all the spaces introduced above are Fréchet spaces. 


\subsection{Pureness and fullness}

\subsection{1. $C^{\infty}$ pure and full almost complex structures}

Definition 2.1. If $S=(1,1)$, or $(2,0),(0,2)$, define

$$
H_{J}^{S}(M)_{\mathbb{R}}=\left\{[\alpha] \in H^{*}(M ; \mathbb{R}) \mid \alpha \in \mathbf{Z}_{J}^{S}\right\}=\frac{\mathbf{Z}_{J}^{S}}{\mathbf{B}_{J}^{S}} .
$$

It is simple to observe

Lemma 2.1. The $J$-compatible cone $\mathcal{K}_{J}^{c}$ is contained in $H_{J}^{1,1}(M)_{\mathbb{R}}$ as an open subset.

Thus it is crucial to understand $H_{J}^{1,1}(M)_{\mathbb{R}}$. Notice that a $J$-tamed symplectic form is a closed smooth 2 -form whose $(1,1)$ component is positive definite, but not necessarily closed.

Remark 2.1. Notice that $\frac{\mathbf{Z}_{J}^{S}}{\mathbf{B}}=\frac{\mathbf{Z}_{J}^{S}}{\mathbf{B} \cap \mathbf{Z}_{S}^{J}}=\frac{\mathbf{Z}_{J}^{S}}{\mathbf{B}_{J}^{S}}$. Thus there is a natural inclusion

$$
\rho_{S}: \frac{\mathbf{Z}_{J}^{S}}{\mathbf{B}_{J}^{S}} \longrightarrow \frac{\mathbf{Z}}{\mathbf{B}}
$$

and

$$
H_{J}^{S}(M)_{\mathbb{R}}=\left\{[\alpha] \in H_{*}(M ; \mathbb{R}) \mid \alpha \in \mathbf{Z}_{J}^{S}\right\}
$$

By abusing notation we will write $\rho_{S}\left(\frac{\mathbf{Z}_{J}^{S}}{\mathbf{B}_{J}^{S}}\right) \subset \frac{\mathbf{Z}}{\mathbf{B}}$ simply as $\frac{\mathbf{Z}_{J}^{S}}{\mathbf{B}_{J}^{S}}$. If there is no confusion we will also write $\Omega^{S}$ for $\Omega_{J}^{S}, \mathbf{Z}^{S}$ for $\mathbf{Z}_{J}^{S}$ and $\mathbf{B}^{S}$ for $\mathbf{B}_{J}^{S}$.

With the notation convention in the above remark, in particular, we have

$$
\frac{\mathbf{Z}^{2}}{\mathbf{B}^{2}} \supset \frac{\mathbf{Z}^{1,1}}{\mathbf{B}^{1,1}}+\frac{\mathbf{Z}^{(2,0),(0,2)}}{\mathbf{B}^{(2,0),(0,2)}}
$$

i.e., $H^{2}(M ; \mathbb{R}) \supset H_{J}^{1,1}(M)_{\mathbb{R}}+H_{J}^{(2,0),(0,2)}(M)_{\mathbb{R}}$.

In this subsection we study when the type decomposition holds for $H^{2}(M ; \mathbb{R})$, i.e.,

$$
H^{2}(M ; \mathbb{R})=H_{J}^{1,1}(M)_{\mathbb{R}} \oplus H_{J}^{(2,0),(0,2)}(M)_{\mathbb{R}}
$$

Definition 2.2. $J$ is said to be $C^{\infty}$ pure if

$$
\frac{\mathbf{Z}^{1,1}}{\mathbf{B}^{1,1}} \cap \frac{\mathbf{Z}^{(2,0),(0,2)}}{\mathbf{B}^{(2,0),(0,2)}}=0 .
$$


Definition 2.3. $J$ is said to be $C^{\infty}$ full if

$$
\frac{\mathbf{Z}}{\mathbf{B}}=\frac{\mathbf{Z}^{1,1}}{\mathbf{B}^{1,1}}+\frac{\mathbf{Z}^{(2,0),(0,2)}}{\mathbf{B}^{(2,0),(0,2)}}
$$

It is immediate from Definition 2.2 and 2.3 that we have

Lemma 2.2. $J$ is $C^{\infty}$ pure and full if and only if we have the type decomposition

$$
H^{2}(M ; \mathbb{R})=H_{J}^{1,1}(M)_{\mathbb{R}} \oplus H_{J}^{(2,0),(0,2)}(M)_{\mathbb{R}}
$$

Let $\pi^{S}: \Omega^{2}(M) \rightarrow \Omega^{S}(M)_{\mathbb{R}}$ be the natural projection. Notice that $\mathbf{B}^{S}=$ $d \Omega^{1}(M) \cap \Omega^{S}(M)_{\mathbb{R}}$ is a proper subspace of $\pi^{S} \mathbf{B}$. In particular, $\pi^{1,1} \mathbf{B}$ is the subspace of $(1,1)$-forms, which are components of exact forms and $\pi^{1,1} \mathbf{Z}$ is the subspace of $(1,1)$-forms, which are components of closed forms.

It is important to understand the quotient spaces

$$
\frac{\pi^{1,1} \mathbf{Z}}{\pi^{1,1} \mathbf{B}}, \quad \frac{\pi^{(2,0),(0,2)} \mathbf{Z}}{\pi^{(2,0),(0,2)} \mathbf{B}}
$$

Since $\mathbf{Z}^{1,1} \subset \pi^{1,1} \mathbf{Z}$ and $\mathbf{B}^{1,1} \subset \pi^{1,1} \mathbf{B}$, there is a natural homomorphism

$$
\iota^{1,1}: \frac{\mathbf{Z}^{1,1}}{\mathbf{B}^{1,1}} \longrightarrow \frac{\pi^{1,1} \mathbf{Z}}{\pi^{1,1} \mathbf{B}}
$$

Similarly there is a homomorphism $\iota^{(2,0),(0,2)}: \frac{\mathbf{Z}^{(2,0),(0,2)}}{\mathbf{B}^{(2,0),(0,2)}} \longrightarrow \frac{\pi^{(2,0),(0,2)} \mathbf{Z}}{\pi^{(2,0),(0,2)} \mathbf{B}}$

Let $W \subset \mathbf{Z}^{2}$ be a subspace lifting $H^{2}(M ; \mathbb{R})$. As mentioned $\mathbf{B}^{2}$ is a closed subspace of $\mathbf{Z}^{2}$, and $W$ is also a closed subspace of $\mathbf{Z}^{2}$ since it is of finite dimensional. So we have a direct sum decomposition

$$
\mathbf{Z}^{2}=\mathbf{B}^{2} \oplus W
$$

In turn, it gives rise to decompositions

$$
\begin{array}{llll}
\pi^{1,1} \mathbf{Z} & =\pi^{1,1} \mathbf{B} & + & \pi^{1,1} W, \\
\pi^{(2,0),(0,2)} \mathbf{Z} & =\pi^{(2,0),(0,2)} \mathbf{B} & + & \pi^{(2,0),(0,2)} W .
\end{array}
$$

Let $\overline{\pi^{1,1} \mathbf{B}}$ be the closure of $\pi^{1,1} \mathbf{B}$ in $\Omega^{1,1}(M)_{\mathbb{R}}$. Notice that $\Omega^{1,1}(M)_{\mathbb{R}}$ is closed in $\Omega^{2}(M)$. So $\overline{\pi^{1,1} \mathbf{B}}$ is the closure of $\pi^{1,1} \mathbf{B}$ in $\Omega^{2}(M)$. Similarly let $\overline{\pi^{1,1} \mathbf{Z}}$ be the closure of $\pi^{1,1} \mathbf{Z}$ in $\Omega^{1,1}(M)_{\mathbb{R}}$. 
There is the natural homomorphism

$$
\phi^{1,1}: \frac{\pi^{1,1} \mathbf{Z}}{\pi^{1,1} \mathbf{B}} \rightarrow \frac{\overline{\pi^{1,1} \mathbf{Z}}}{\overline{\pi^{1,1} \mathbf{B}}} .
$$

Similarly there is the homomorphism $\phi^{(2,0),(0,2)}$ for $(2,0),(0,2)$ forms.

Lemma 2.3. $\phi^{1,1}$ and $\phi^{(2,0),(0,2)}$ are surjective.

Proof. Notice that $\pi^{1,1} W$ is a finite dimensional space. We use the fact on page 22 in [26].

Lemma 2.4. Let $V$ be a topological vector space over $\mathbb{R}$. If $Q$ is a closed subspace of $V$ and $P$ is a finite dimensional subspace of $V$, then $Q+P$ is closed in $V$.

Proof. Let $t: V \rightarrow V / Q$ be the quotient map. Since $Q$ is closed, the quotient $V / Q$ is Hausdorff. Thus $t(P)$ is also Hausdorff. Since $t(P)$ is also finite dimensional subspace of $V / Q$, as a topological space $t(P)$ is isomorphic to $\mathbb{R}^{k}$ for some integer $k$ by Theorem 3.2 in [26]. Thus $t(P)$ is complete in the sense that each Cauchy filter converges. Therefore $t(P)$ is closed in $V / Q$. (For general $P$, even $P$ is closed in $V$, it is not necessary that the image $t(P)$ is closed in $V / Q)$. Finally, since $t$ is continuous, $Q+P=t^{-1}(t(P))$ is a closed subspace of $V$.

Let $\tilde{W}^{1} \subset \pi^{1,1} W$ be a subspace mapping isomorphically to $\frac{\pi^{1,1} \mathbf{Z}}{\pi^{1,1} \mathrm{~B}}$, and similarly define $\tilde{W}^{2} \subset \pi^{(2,0),(0,2)} W$. Then we still have $(2.2)$ with $\tilde{W}^{1}$ and $\tilde{W}^{2}$.

Apply Lemma 2.4 to the case

$$
\begin{aligned}
& V=\Omega^{1,1}(M)_{\mathbb{R}} \quad\left(\Omega^{(2,0),(0,2)}(M)_{\mathbb{R}}\right), \\
& Q=\overline{\pi^{1,1} \mathbf{B}} \quad\left(\overline{\pi^{(2,0),(0,2)} \mathbf{B}}\right), \quad P=\tilde{W}^{1}\left(\tilde{W}^{2}\right)
\end{aligned}
$$

to obtain

$$
\begin{array}{ll}
\overline{\pi^{1,1} \mathbf{Z}} & =\overline{\pi^{1,1} \mathbf{B}}+\tilde{W}^{1}, \\
\overline{\pi^{(2,0),(0,2)} \mathbf{Z}} & =\overline{\pi^{(2,0),(0,2)} \mathbf{B}}+\tilde{W}^{2} .
\end{array}
$$

Therefore $\tilde{W}^{1}\left(\tilde{W}^{2}\right)$ projects surjectively onto

$$
\frac{\overline{\pi^{1,1} \mathbf{Z}}}{\overline{\pi^{1,1} \mathbf{B}}} \quad\left(\frac{\overline{\pi^{(2,0),(0,2)} \mathbf{Z}}}{\overline{\pi^{(2,0),(0,2)} \mathbf{B}}}\right) .
$$


Lemma 2.5. $J$ is $C^{\infty}$ pure if and only if

$$
\pi^{1,1} \mathbf{B} \cap \mathbf{Z}^{1,1}=\mathbf{B}^{1,1},
$$

and (2.4) is equivalent to

$$
\pi^{(2,0),(0,2)} \mathbf{B} \cap \mathbf{Z}^{(2,0),(0,2)}=\mathbf{B}^{(2,0),(0,2)} .
$$

Consequently, $J$ being $C^{\infty}$ pure is equivalent to $\iota^{1,1}$ being injective, which is also equivalent to $\iota^{(2,0),(0,2)}$ being injective.

Proof. The equivalence between (2.4) and (2.5) follows from that $\pi^{1,1} d \gamma$ is closed (exact) if and only if $\pi^{(2,0),(0,2)} d \gamma$ is closed (exact).

Suppose (2.4) is true. We want to prove that $J$ is $C^{\infty}$ pure, i.e., if $e \in \mathbf{Z}^{1,1}, f \in \mathbf{Z}^{(2,0),(0,2)}$ and $[e]=[f] \in H^{2}(M ; \mathbb{R})$, then $[e]=[f]=0$.

Since $e-f \in \mathbf{B}, \pi^{1,1}(e-f)=e \in \pi^{1,1} \mathbf{B}$. As $e \in \mathbf{Z}^{1,1}$, we conclude that $e \in \mathbf{B}^{1,1}$ and hence $[e]=[f]=0$. This proves that $J$ is pure.

Conversely, suppose $J$ is pure. We need to show that if $e \in \mathbf{Z}^{1,1}$ and $e=\pi^{1,1} d \gamma$, then $[e]=0$.

Let $-f=d \gamma-e$. Then $d f=0$ and thus $f \in \mathbf{Z}^{(2,0),(0,2)}$. Since $[e-f]=0$ we conclude that $[e]=0$.

Remark 2.2. It is not clear we can replace $\pi^{1,1} \mathbf{B}$ in Lemmas 2.5 by its closure $\overline{\pi^{1,1} \mathbf{B}}$.

Suppose $J$ is $C^{\infty}$ full. Then we can choose $W=W^{1} \oplus W^{2}$ with $W^{1} \subset$ $\mathbf{Z}^{1,1}$ a subspace lifting $H_{J}^{1,1}(M)_{\mathbb{R}}$, and $W^{2} \subset \mathbf{Z}^{(2,0),(0,2)}$ a subspace lifting $H_{J}^{(2,0),(0,2)}(M)_{\mathbb{R}}$. Then $\pi^{1,1} W=W^{1}$ and $\pi^{(2,0),(0,2)} W=W^{2}$. This proves

Lemma 2.6. If $J$ is $C^{\infty}$ full then both $\iota^{1,1}$ and $\iota^{(2,0),(0,2)}$ are surjective. Consequently, if $J$ is $C^{\infty}$ pure and full, then both $\iota^{1,1}$ and $\iota^{(2,0),(0,2)}$ are isomorphisms.

Notice that when $J$ is integrable, there is the Dolbeault complex and the associated Dolbeault cohomology groups. But our groups are subgroups of the De Rham homology and cohomology groups, and might be different from the Dolbeault groups. Nonetheless, there is the following identification.

Proposition 2.1 [11]. Let $J$ be a complex structure on a 2 -manifold. If $J$ is Kähler or $n=2$ then $J$ is $C^{\infty}$ pure and full. Moreover,

$$
\begin{array}{ll}
H_{J}^{1,1}(M)_{\mathbb{R}} & =H_{\bar{\partial}}^{1,1}(M)_{\mathbb{R}} \\
H_{J}^{(2,0),(0,2)}(M)_{\mathbb{R}} & =\left(H_{\bar{\partial}}^{2,0}(M) \oplus H_{\bar{\partial}}^{0,2}(M)\right)_{\mathbb{R}} .
\end{array}
$$


Remark 2.3. It is interesting to investigate whether some non-integrable $J$ could be $C^{\infty}$ pure and full.

It is shown in $[11,12]$ that if $J$ is almost Kähler, then it is $C^{\infty}$ pure. While in [12] a left-invariant almost complex structure on a 6 -dimensional nilmanifold is found to be not $C^{\infty}$ pure.

In [11] it is shown that every almost complex structure on a 4-dimensional manifold is $C^{\infty}$ pure and full. Some higher dimensional examples are also given in [12].

In addition, in dimension 4 , the groups

$$
H_{J}^{1,1}(M)_{\mathbb{R}}, \quad H_{J}^{(2,0),(0,2)}(M)_{\mathbb{R}}
$$

are determined in [11] for any $J$ metric related to an integrable one (i.e. when there is a metric $g$ which is compatible with $J$ and an integrable almost complex structure at the same time), and their dimensions are estimated for those $J$ tamed by symplectic forms.

\subsubsection{Pure and full almost complex structures}

Definition 2.4. If $S=(1,1)$ or $(2,0),(0,2)$, define

$$
H_{S}^{J}(M)_{\mathbb{R}}=\frac{\mathcal{Z}_{S}^{J}}{\mathcal{B}_{S}^{J}}
$$

We will adopt similar notation convention as in Remark 2.1.

Definition 2.5. $J$ is said to be pure if

$$
\frac{\mathcal{Z}_{1,1}}{\mathcal{B}_{1,1}} \cap \frac{\mathcal{Z}_{(2,0),(0,2)}}{\mathcal{B}_{(2,0),(0,2)}}=0 .
$$

$J$ is said to be full if

$$
\frac{\mathcal{Z}_{2}}{\mathcal{B}_{2}}=\frac{\mathcal{Z}_{1,1}}{\mathcal{B}_{1,1}}+\frac{\mathcal{Z}_{(2,0),(0,2)}}{\mathcal{B}_{(2,0),(0,2)}} .
$$

Clearly, the analogue of Lemma 2.2 still holds.

Lemma 2.7. $J$ is pure and full if and only if we have the type decomposition

$$
H_{2}(M ; \mathbb{R})=H_{1,1}^{J}(M)_{\mathbb{R}} \oplus H_{(2,0),(2,0)}^{J}(M)_{\mathbb{R}} .
$$

One can follow the proof of Proposition 2.1 in [11] and work with dual complex and dual operations to prove (see also Remark 2.4). 
Proposition 2.2. Let $J$ be a complex structure on a $2 n$-manifold. If $J$ is Kähler or $n=2$, then it is pure and full.

Let $\pi_{S}: \mathcal{E}_{2}(M) \rightarrow \mathcal{E}_{S}(M)_{\mathbb{R}}$ be the natural projection. Notice that $\mathcal{B}_{S}=$ $d \mathcal{E}_{3}(M) \cap \mathcal{E}_{S}(M)_{\mathbb{R}}$ is a proper subspace of $\pi_{S} \mathcal{B}$. In particular, $\pi_{1,1} \mathcal{B}$ is the subspace of bidimension $(1,1)$ currents that are components of boundaries and $\pi_{1,1} \mathcal{Z}$ is the subspace of bidimension $(1,1)$ currents that are components of cycles.

It is also important to understand the quotient spaces

$$
\frac{\pi_{1,1} \mathcal{Z}}{\pi_{1,1} \mathcal{B}}, \quad \frac{\pi_{(2,0),(0,2)} \mathcal{Z}}{\pi_{(2,0),(0,2)} \mathcal{B}}
$$

Consider the natural homomorphisms

$$
\iota_{1,1}: \frac{\mathcal{Z}_{1,1}}{\mathcal{B}_{1,1}} \longrightarrow \frac{\pi_{1,1} \mathcal{Z}}{\pi_{1,1} \mathcal{B}}, \quad \iota_{(2,0),(0,2)}: \frac{\mathcal{Z}_{(2,0),(0,2)}}{\mathcal{B}_{(2,0),(0,2)}} \longrightarrow \frac{\pi_{(2,0),(0,2)} \mathcal{Z}}{\pi_{(2,0),(0,2)} \mathcal{B}}
$$

Lemma 2.8. $J$ being pure is equivalent to $\iota_{1,1}$ being injective, which is also equivalent to $\iota_{(2,0),(0,2)}$ being injective.

If $J$ is full then both $\iota_{1,1}$ and $\iota_{(2,0),(0,2)}$ are surjective.

Consequently, if $J$ is pure and full, then both $\iota_{1,1}$ and $\iota_{(2,0),(0,2)}$ are isomorphisms.

Of course we also have the homomorphisms $\phi_{1,1}$ and $\phi_{(2,0),(0,2)}$, and

Lemma 2.9. $\phi_{1,1}$ and $\phi_{(2,0),(0,2)}$ are surjective.

2.2.3. Closed almost complex structures To compare homological properties of $J$ for the complexes of currents and forms we further introduce the following condition.

Definition 2.6. An almost complex structure $J$ is said to be closed if $\pi_{1,1} \mathcal{B}$ is a closed subspace of $\mathcal{E}_{1,1}(M)_{\mathbb{R}}$.

$J$ is said to be $C^{\infty}$ closed if the image of the operator

$$
d: \Omega^{1,1}(M)_{\mathbb{R}} \rightarrow \Omega^{3}(M)
$$

is a closed subspace of $\Omega^{3}(M)$. 
Notice that $\pi_{1,1} \mathcal{B}$ is the image of the operator

$$
\pi_{1,1} d: \mathcal{E}_{3}(M) \rightarrow \mathcal{E}_{1,1}(M)_{\mathbb{R}}
$$

The adjoint of this operator $\pi_{1,1} d$ can be easily computed to be the operator (2.8). Recall that the closed range theorem says if a linear map between Fréchet spaces has closed range then its adjoint also has closed range. Thus we have

Lemma 2.10. $J$ is closed if and only if $J$ is $C^{\infty}$ closed.

By the same argument for Lemma 2.3, we have

Lemma 2.11. If $J$ is a closed almost complex structure, then $\pi_{1,1} \mathcal{Z}$ is also a closed subspace of $\mathcal{E}_{1,1}(M)_{\mathbb{R}}$.

Notice that if $J$ is a complex structure, then the image of the operator $(2.8)$ actually lies in $\Omega^{(2,1),(1,2)}(M)_{\mathbb{R}}:=\left(\Omega_{J}^{2,1}(M)_{\mathbb{C}} \oplus \Omega_{J}^{1,2}(M)_{\mathbb{C}}\right) \cap \Omega^{3}(M)$. And it was shown in [16] that in this case the image is a closed subspace of $\Omega^{(2,1),(1,2)}(M)_{\mathbb{R}}$. Denote this image by $V$. We write

$$
V=\left(\pi^{(2,1),(1,2)}\right)^{-1}(V) \cap\left(\pi^{(3,0),(0,3)}\right)^{-1}(0),
$$

where $\pi^{(2,1),(1,2)}$ and $\pi^{(3,0),(0,3)}$ are projection operators from $\Omega^{3}(M)$ to $\Omega^{(2,1),(1,2)}(M)_{\mathbb{R}}$ and $\Omega^{(3,0),(0,3)}(M)_{\mathbb{R}}:=\left(\Omega_{J}^{3,0}(M)_{\mathbb{C}} \oplus \Omega_{J}^{0,3}(M)_{\mathbb{C}}\right) \cap \Omega^{3}(M)$, respectively. By the continuity of $\pi^{(2,1),(1,2)}$ and $\pi^{(3,0),(0,3)}$, we see that $V$ is also closed in $\Omega^{3}(M)_{\mathbb{R}}$. Thus we conclude

Proposition 2.3. Any complex structure is closed (and also $C^{\infty}$ closed).

It would be interesting to see whether the explicit deformation $J_{\alpha}$ of a complex structure $J$ by a holomorphic 2 -form $\alpha$ in [18] is closed.

\subsection{Duality}

In this subsection we compare $H_{1,1}^{J}(M)_{\mathbb{R}}$ and $H_{J}^{1,1}(M)_{\mathbb{R}}$. We recall the following well-known fact.

Lemma 2.12. A real $(1,1)$-form is closed if and only if it vanishes on $\pi_{1,1} \mathcal{B}$, and a real $(1,1)$-form is exact if and only if it vanishes on $\pi_{1,1} \mathcal{Z}$. The same is true in the $(2,0),(0,2)$ setting. 
Proof. Suppose $\alpha$ is a real closed $(1,1)$-form. Then for any $u \in \mathcal{E}(M)_{\mathbb{R}}$, we have

$$
\alpha \cdot \pi_{1,1}(d u)=\alpha \cdot d u=d \alpha \cdot u=0
$$

i.e., $\alpha$ vanishes on $\pi_{1,1} \mathcal{B}$. Conversely, if $\alpha$ vanishes on $\pi_{1,1} \mathcal{B}$, then we still have (2.9). This implies that $d \alpha=0$ since forms are dual to currents.

Suppose $d \beta$ is a real exact $(1,1)$-form, then for any $v \in \mathcal{Z}$, we have

$$
d \beta \cdot \pi_{1,1} v=d \beta \cdot v=\beta \cdot d v=0,
$$

i.e., $d \beta$ vanishes on $\pi_{1,1} \mathcal{Z}$. For the converse statement, suppose $\gamma$ is a real $(1,1)$-form vanishing on $\pi_{1,1} \mathcal{Z}$. Then $\gamma$ vanishes on $\mathcal{Z}$. By Theorem $17^{\prime}$ in [6], $\gamma$ is exact.

Since a real $(1,1)$-form vanishes on $\pi_{1,1} \mathcal{B}\left(\pi_{1,1} \mathcal{Z}\right)$ if and only if it vanishes on $\overline{\pi_{1,1} \mathcal{B}}\left(\overline{\pi_{1,1} \mathcal{Z}}\right)$, we have

Lemma 2.13. A real (1,1)-form is closed if and only if it vanishes on $\overline{\pi_{1,1} \mathcal{B}}$, and a real $(1,1)$-form is exact if and only if it vanishes on $\overline{\pi_{1,1} \mathcal{Z}}$. The same is true in the $(2,0),(0,2)$ setting.

The following is a crucial observation based on Lemma 2.13 and the Hahn-Banach Theorem.

Proposition 2.4. For any almost complex structure J, there is a natural isomorphism

$$
\bar{\sigma}^{1,1}: H_{J}^{1,1}(M)_{\mathbb{R}}=\frac{\mathbf{Z}^{1,1}}{\mathbf{B}^{1,1}} \rightarrow\left(\frac{\overline{\pi_{1,1} \mathcal{Z}}}{\overline{\pi_{1,1} \mathcal{B}}}\right)^{*}
$$

Similarly, there are natural isomorphisms $\bar{\sigma}^{(2,0),(0,2)}, \bar{\sigma}_{1,1}, \bar{\sigma}_{(2,0),(0,2)}$.

Proof. By Lemma 2.13, closed $(1,1)$-forms vanish on $\overline{\pi_{1,1} \mathcal{B}}$, thus there is a homomorphism $\sigma^{\prime}: \mathbf{Z}^{1,1} \rightarrow\left(\frac{\overline{\pi_{1,1} \mathcal{Z}}}{\overline{\pi_{1,1} \mathcal{B}}}\right)^{*}$. The kernel of $\sigma^{\prime}$ is $\mathbf{B}^{1,1}$ by Lemma 2.12 . Therefore $\bar{\sigma}^{1,1}$ is well-defined and an injection.

The surjectivity is proved by an application of the Hahn-Banach Theorem to construct a homomorphism in the reverse direction. An element in $\left(\frac{\overline{\pi_{1,1} \mathcal{Z}}}{\overline{\pi_{1,1} \mathcal{B}}}\right)^{*}$ is the same as a functional $L$ on $\overline{\pi_{1,1} \mathcal{Z}}$ vanishing on $\overline{\pi_{1,1} \mathcal{B}}$. Since $\overline{\pi_{1,1} \mathcal{Z}}$ is closed subspace of $\mathcal{E}_{1,1}(M)_{\mathbb{R}}$, we can extend $L$ to a functional $\mathcal{L}$ on $\mathcal{E}_{1,1}(M)_{\mathbb{R}}$ vanishing on $\overline{\pi_{1,1} \mathcal{B}}$. Such a $\mathcal{L}$ corresponds to a closed $(1,1)$-form by 
Lemma 2.12. If $\mathcal{L}^{\prime}$ is another extension of $L$, then $\mathcal{L}^{\prime}-\mathcal{L}$ vanishes on $\overline{\pi_{1,1} \mathcal{Z}}$ by Lemma 2.12. And since We have shown that there is a homomorphism $\tau:\left(\frac{\overline{\pi_{1,1} \mathcal{Z}}}{\pi_{1,1} \mathcal{B}}\right)^{*} \rightarrow H_{J}^{1,1}(M)_{\mathbb{R}}$. Since $\bar{\sigma}^{1,1} \tau=\mathrm{id}, \bar{\sigma}^{1,1}$ is also surjective.

If $J$ is closed, by Lemma $2.11, \bar{\sigma}^{1,1}$ becomes the isomorphism

$$
\sigma^{1,1}: H_{J}^{1,1}(M)_{\mathbb{R}}=\frac{\mathbf{Z}^{1,1}}{\mathbf{B}^{1,1}} \rightarrow\left(\frac{\pi_{1,1} \mathcal{Z}}{\pi_{1,1} \mathcal{B}}\right)^{*}
$$

Together with Lemma 2.8, we obtain

Corollary 2.1. Let $J$ be a closed and pure almost complex structure. Then we have

$$
\operatorname{dim} H_{J}^{1,1}(M)_{\mathbb{R}} \geq \operatorname{dim} H_{1,1}^{J}(M)_{\mathbb{R}}
$$

If $J$ is also full, then $H_{1,1}^{J}(M)_{\mathbb{R}}$ and $H_{J}^{1,1}(M)_{\mathbb{R}}$ have the same dimension and are dual to each other.

By Lemmas 2.3, 2.9, and Proposition 2.4, we have

Corollary 2.2. Suppose $\iota^{1,1}\left(\iota^{(2,0),(0,2)}\right)$ is surjective, then

$$
\begin{aligned}
\operatorname{dim} H_{J}^{1,1}(M)_{\mathbb{R}} & \geq \operatorname{dim} H_{1,1}^{J}(M)_{\mathbb{R}}\left(\operatorname{dim} H_{J}^{(2,0),(0,2)}(M)_{\mathbb{R}}\right. \\
& \left.\geq \operatorname{dim} H_{(2,0),(0,2)}^{J}(M)_{\mathbb{R}}\right)
\end{aligned}
$$

Suppose $\iota_{1,1}\left(\iota_{(2,0),(0,2)}\right)$ is surjective, then the reverse inequality holds.

By Lemmas 2.6 and 2.8 and Corollary 2.2, we obtain

Corollary 2.3. Suppose $J$ is $C^{\infty}$ full, then

$$
\begin{array}{ll}
\operatorname{dim} H_{J}^{1,1}(M)_{\mathbb{R}} & \geq \operatorname{dim} H_{1,1}^{J}(M)_{\mathbb{R}}, \\
\operatorname{dim} H_{J}^{(2,0),(0,2)}(M)_{\mathbb{R}} & \geq \operatorname{dim} H_{(2,0),(0,2)}^{J}(M)_{\mathbb{R}} .
\end{array}
$$

Suppose $J$ is full, then the reverse inequalities hold.

Notice that $H_{1,1}^{J}(M)_{\mathbb{R}}$ pairs trivially with $H_{J}^{(2,0),(0,2)}(M)_{\mathbb{R}}$, and $H_{J}^{1,1}(M)_{\mathbb{R}}$ pairs trivially with $H_{(2,0),(0,2)}^{J}(M)_{\mathbb{R}}$. Suppose $e \in H_{1,1}^{J}(M)_{\mathbb{R}} \cap H_{(2,0),(0,2)}^{J}$ $(M)_{\mathbb{R}}$. Then $e$ pairs trivially with $H_{J}^{1,1}(M)_{\mathbb{R}}+H_{J}^{(2,0),(0,2)}(M)_{\mathbb{R}}$. Since the pairing of $H_{2}(M ; \mathbb{R})$ and $H^{2}(M ; \mathbb{R})$ is non-degenerate, we have 
Proposition 2.5. If $J$ is $C^{\infty}$ full, then it is pure and we have (2.13). Similarly, if $J$ is full, then it is $C^{\infty}$ pure and the reverse inequalities hold.

In particular, if $J$ is both full and $C^{\infty}$ full, then it is pure and full, as well as $C^{\infty}$ pure and full.

If $J$ is pure and full as well as $C^{\infty}$ pure and full, then the J-homology and cohomology decompositions of $H_{2}(M ; \mathbb{R})$ and $H^{2}(M ; \mathbb{R})$ are dual to each other.

Remark 2.4. It is observed in [12] that if $J$ is $C^{\infty}$ pure and full, then it is pure. Fino and Tomassini further show that if $J$ is a $C^{\infty}$ pure and full almost complex structure on a $2 n$-dimensional manifold, then $J$ is also pure and full under any of the following three conditions:

- $n=2$, or

- there is a metric $g$ compatible with $J$ such that any cohomology class in $H_{J}^{1,1}(M)_{\mathbb{R}}\left(H_{J}^{(2,0),(0,2)}(M)_{\mathbb{R}}\right)$ has a $g$-harmonic representative in $\mathcal{Z}_{J}^{1,1}$ $\left(\mathcal{Z}^{(2,0),(0,2)}\right)$ respectively, or

- $J$ is compatible with a symplectic form $\omega$ of Lefschetz type.

The first condition implies that when $n=2$ any $J$ is pure and full (see Remark 2.3), while the first and the third conditions imply Proposition 2.2. Many families of pure and full (non-Kähler) almost complex structures on compact nilmanifolds and solvmanifolds are also constructed this way in [12].

\section{Symplectic forms versus complex cycles}

\subsection{Complex cycles and tamed symplectic forms}

In this subsection we describe Sullivan's approach to tamed symplectic forms.

3.1.1. Structure cycles and closed transversal forms Let us first review some general basic concepts in [28]. A compact convex cone $\mathcal{C}$ in a locally convex topological space over $\mathbb{R}$ is a convex cone which for some continuous linear functional $L$ satisfies $L(w)>0$ for $w \neq 0$ in $\mathcal{C}$ and $L^{-1}(1) \cap$ $\mathcal{C}$ is compact. The latter set is called the base for the cone.

A cone structure (of 2-directions) on $M$ is a continuous field of compact convex cones $\left\{\mathcal{C}_{x}\right\}$ in the vector space $\Lambda_{2}(x)=\Lambda^{2} T_{x} M$ of tangent 2-vectors on $M$. Such a cone structure is called ample if at each point $x$ the cone $\mathcal{C}_{x}$ intersects the linear span of the Schubert variety $S_{\tau}$ of every 2-plane $\tau$ at $x$ ( $S_{\tau}$ is the set of 2-planes that intersect $\tau$ in at least a line). 
A smooth 2 -form $\omega$ on $M$ is transversal to the cone structure $\mathcal{C}$ if $\omega(v)>0$ for each $v \neq 0$ in $\mathcal{C}_{x} \subset \Lambda_{2}(x), x \in M$. Using a partition of unity it is easy to see that such transversal forms always exist.

A Dirac current is the one determined by the evaluation of 2 -forms on a single 2 -vector at one point. The cone of structure currents $\mathfrak{C}$ associated to the cone structure $\mathcal{C}$ is the closed convex cone of currents generated by the Dirac currents associated to the elements of $\mathcal{C}_{x}, x \in M$. It is easy to see that if $M$ is compact then $\mathfrak{C}$ is a compact convex cone.

The structure cycles of $\mathcal{C}$ are the structure currents of $\mathcal{C}$ which are closed as currents. Let $Z \mathfrak{C}$ be the cone of structure cycles and let $H \mathfrak{C}$ be the cone of homology classes of structure cycles in $H_{2}(M ; \mathbb{R})$. Let $\breve{H C} \subset H^{2}(M ; \mathbb{R})$ be the dual cone defined by $(\breve{H \mathfrak{C}}, H \mathfrak{C}) \geq 0$. Notice that the interior of $\breve{H \mathfrak{C}}$ is characterized by $(\operatorname{Int} \breve{H C}, H \mathfrak{C})>0$ when $H \mathfrak{C} \neq\{0\}$. That is because if we have an element $a$ in the interior of $\breve{H C}$, pair with some element $s \in H \mathfrak{C}$ is 0 , then there exists $a^{\prime}$ such that $\left(a^{\prime}, s\right)<0$. Thus $\left(a+t a^{\prime}, s\right)<0$ for small positive $t$. That is a contradiction.

Let $\mathcal{E}_{p}(M)_{\mathbb{R}}$ be the space of real $p$-dimensional currents. Let $\mathcal{B} \subset \mathcal{E}_{2}(M)_{\mathbb{R}}$ be the subspace of boundaries and $\mathcal{Z} \subset \mathcal{E}_{2}(M)_{\mathbb{R}}$ be the subspace of cycles.

Sullivan made the following beautiful observation using the duality between forms and currents, the Hahn-Banach theorem, and the compactness.

Theorem $3.1[\mathbf{2 8}]$. Let $\mathcal{C}$ be an ample cone structure. Then $\mathcal{C}$ has nontrivial cycles. And if no non-trivial structure cycle is homologous to zero, i.e.,

$$
Z \mathfrak{C} \cap \mathcal{B}=\{0\},
$$

then $M$ admits a closed 2-form transverse to the ample 2-direction structure. Moreover, $H \mathfrak{C}$ is a compact convex cone and the interior of $\breve{H C} \subset H^{2}(M ; \mathbb{R})$ consists precisely of the classes of closed forms transverse to $\mathcal{C}$.

3.1.2. Complex cycles Let $J$ be an almost complex structure on $M$. Let $\mathcal{C}(J)$ be the cone structure of complex lines. As for any plane $\tau$ and any nonzero vector $v$ in $\tau$ the complex line $(v, J v)$ intersects $\tau$ at least in $v, \mathcal{C}(J)$ is an ample cone structure.

In particular, a 2-dimensional current is of bidimension $(1,1)$ if it can be locally expressed as

$$
T=\sum_{j, k} T^{j k} \frac{\sqrt{-1}}{2} X_{j} \wedge \bar{X}_{k},
$$


where $T^{j k}$ is a distribution and $\left\{X_{1}, X_{2}, \ldots\right\}$ is a basis of type $(1,0)$ vectors. Such a current $T$ is said to be positive if $\sum T^{j k} w_{j} \bar{w}_{k}$ is a non-negative measure for each $w \in \mathbb{C}^{n}$.

Lemma 3.1 [16]. A structure current associated to $\mathcal{C}(J)$ is a positive current of bidimension $(1,1)$.

The argument is as follows. Firstly, there exists a non-negative Radon measure $\|T\|$ called the total variation measure of $T$ and a 2 -vector $\vec{T}$, which is $\|T\|$ measurable, such that $T=\|T\| \vec{T}$. Secondly, a 2-dimensional current $T$ is positive if and only if $\vec{T}(x)$ belongs to $\mathcal{C}(J)_{x}$ for each $x \in M$.

In this case a structure cycle is called a complex cycle.

More generally, we can consider the cone of complex cycles of $\mathbb{C}$ dimension $p$ and denote it by $C_{p}$. In particular, $C_{1}=Z \mathfrak{C}(J)$. Let $D_{p} \subset C_{p}$ denote the subcone of diffuse complex cycles, which consists of currents in $C_{p}$ given by closed $2 n-2 p$ forms.

Lemma 3.2 [28]. A J-compatible form $\alpha$ gives rises to a diffuse complex cycle in $D_{n-1}$. Conversely, a diffuse cycle in $D_{n-1}$ whose interior support is all of $M$ is a J-compatible form.

To illustrate why this is true we look at the case $n=2$. In a basis $\left\{z_{1}=\right.$ $\left.x_{1}+\sqrt{-1} y_{1}, z_{2}=x_{2}+\sqrt{-1} y_{2}\right\}$ of $\mathbb{C}^{2}$ the canonical form

$$
d x_{1} \wedge d y_{1}+d x_{2} \wedge d y_{2}
$$

on $\mathbb{R}^{4}$ corresponds to the ray given by the sum of the coordinate complex lines

$$
\left\{\frac{\partial}{\partial x_{1}}, \frac{\partial}{\partial y_{1}}=\sqrt{-1} \frac{\partial}{\partial x_{1}}\right\} \quad \text { and } \quad\left\{\frac{\partial}{\partial x_{2}}, \frac{\partial}{\partial y_{2}}=\sqrt{-1} \frac{\partial}{\partial x_{2}}\right\}
$$

of $\mathbb{C}^{2}$ in $\Lambda_{2}$.

It was also observed in [28] that the natural intersection pairing between forms and currents satisfies $D_{p} \cdot C_{q} \subset C_{p+q-n}$. In particular, the diffuse complex cycles form a semi-ring under intersection. This implies that if $\alpha_{i}, 1 \leq$ $i \leq n$ are $n J$-compatible forms, then $\prod_{i=1}^{n} \alpha_{i}>0$. This is true because, at each point $x$, the form $\wedge_{i=1}^{n} \alpha_{i}>0$ is positive on a complex basis of the form $\left\{v_{i}, J v_{i}\right\}$.

Finally, we write down the geometric part of $H \mathfrak{C}(J)$ for a complex structure $J$. When $J$ is a complex structure, a $p$-dimensional analytic subset of 
$M$ is in $\mathbb{C}_{p}$. Hence there are the following geometric objects in $Z \mathfrak{C}(J)$ : If $Y$ is a $p$-dimensional analytic subset of $M$ and $\omega$ is a Kähler form, then $Y \wedge \omega^{p-1} \in Z \mathfrak{C}(J)$. Define

$$
T=\operatorname{PD}\left(\left(\mathcal{K}_{J}^{c}\right)^{n-1}\right)+H C \mathfrak{Y}^{1} \cdot \operatorname{PD}\left(\left(\mathcal{K}_{J}^{c}\right)^{n-2}\right)+\cdots+H C \mathfrak{Y}^{n-1},
$$

where $H C \mathfrak{Y}^{i} \subset H_{J}^{i, i}(M)_{\mathbb{R}}$ is the cone generated by classes of $(n-i)$ dimensional irreducible analytic subsets of $M . T \subset H \mathfrak{C}(J)$ is considered to be the geometric part of $H \mathfrak{C}(J)$.

We might be able to define the analogue of $T$ for a general almost complex structure replacing analytic subsets by either the zero sets or the images of locally pseudo-holomorphic maps.

3.1.3. Tamed symplectic forms Notice that a closed form transverse to $\mathcal{C}(J)$ is nothing but a $J$-tamed symplectic form. As an almost symplectic form on a closed manifold cannot be exact, it was observed in [28] that the cone of complex cycles $Z \mathfrak{C}(J)$ is non-empty. Moreover, $Z \mathfrak{C}(J)$ is a compact convex cone in the space of 2 -currents. As a consequence of Theorem 3.1 we have

Theorem 3.2 [28]. Let $(M, J)$ be an almost complex $2 n$-manifold. Then $\mathcal{K}_{J}^{t}$ is non-empty if and only if there is no non-trivial positive current of bidimension $(1,1)$ which is a boundary. Moreover, under the (non-degenerate) pairing between $H^{2}(M ; \mathbb{R})$ and $H_{2}(M ; \mathbb{R}), \mathcal{K}_{J}^{t} \subset H^{2}(M ; \mathbb{R})$ is the interior of the dual cone of $H \mathfrak{C}(J) \subset H_{2}(M ; \mathbb{R})$.

\subsection{Complex cycles and compatible symplectic forms}

Notice that a 2 -form $\omega$ is a $J$-compatible symplectic form if and only if it is of type $(1,1)$, closed and tamed by $J$.

A $(1,1)$-form corresponds to a functional on $\mathcal{E}_{1,1}(M)_{\mathbb{R}}$. By Lemma 2.12 a functional on $\mathcal{E}_{1,1}(M)_{\mathbb{R}}$ gives rise to a closed form if and only if it vanishes on $\pi_{1,1} \mathcal{B}$. By definition, a functional on $\mathcal{E}_{1,1}(M)_{\mathbb{R}}$ gives rises to a $J$-tamed form if and only if it is positive on $\mathcal{C}(J) \backslash\{0\}$. Thus we have

Lemma 3.3. There is a bijection between $J$-compatible symplectic forms and functionals on $\mathcal{E}_{1,1}(M)_{\mathbb{R}}$ vanishing on $\overline{\pi_{1,1} \mathcal{B}}$ and positive on $\mathcal{C}(J) \backslash\{0\}$.

When $J$ is integrable there is the following Kähler criterion. 
Theorem 3.3 [16]. Suppose $(M, J)$ is a compact complex manifold. Then there exists a Kähler metric for $M$ if and only if

$$
\mathfrak{C}(J) \cap \pi_{1,1} \mathcal{B}=0 .
$$

By Lemma 3.3, for any almost Kähler complex structure, (3.2) is replaced by

$$
\mathfrak{C}(J) \cap \overline{\pi_{1,1} \mathcal{B}}=0 .
$$

We can further characterize $\mathcal{K}_{J}^{c}$.

Theorem 3.4. Suppose $(M, J)$ is an almost complex manifold. If $\mathcal{K}_{J}^{c}$ is non-empty then $\mathcal{K}_{J}^{c} \subset H_{J}^{1,1}(M)_{\mathbb{R}}$ is the interior of the dual cone of $H \mathfrak{C}(J) \subset$ $H_{1,1}^{J}(M)_{\mathbb{R}}$.

Proof. First of all, under the (possibly degenerate) pairing between $H_{J}^{1,1}(M)_{\mathbb{R}}$ and $H_{1,1}^{J}(M)_{\mathbb{R}}, \mathcal{K}_{J}^{c}$ is contained in the interior of the dual cone of $H \mathfrak{C}(J)$ by the definition of the positive current and Lemma 3.1.

It remains to prove that if $e \in H_{J}^{1,1}(M)_{\mathbb{R}}$ is positive on $H \mathfrak{C}(J)$, then it is represented by a $J$-compatible form. Consider the element $\bar{\sigma}(e)$ in $\left(\frac{\overline{\pi_{1,1} \mathcal{Z}}}{\pi_{1,1} \mathcal{B}}\right)^{*}$, which pulls back to a functional $L$ on $\overline{\pi_{1,1} \mathcal{Z}}$ vanishing on $\overline{\pi_{1,1} \mathcal{B}}$. Denote the kernel hyperplane of $L$ in $\overline{\pi_{1,1} \mathcal{Z}}$ also by $L$. By our choice of $e$, as subsets of $\mathcal{E}_{1,1}(M)_{\mathbb{R}}, L$ and $\mathfrak{C}(J) \backslash\{0\}$ are disjoint.

Since $\overline{\pi_{1,1} \mathcal{Z}}$ is a closed subspace of $\mathcal{E}_{1,1}(M)_{\mathbb{R}}$, any kernel hyperplane in $\overline{\pi_{1,1} \mathcal{Z}}$ containing $\overline{\pi_{1,1} \mathcal{B}}$ is also a closed subspace of $\mathcal{E}_{1,1}(M)_{\mathbb{R}}$.

Choose a Hermitian metric $h$ and let $\psi$ be the associated real $(1,1)$ form. Set

$$
\overline{\mathfrak{C}}(J)=\{T \in \mathfrak{C}(J) \mid T(\psi)=1\} .
$$

It was shown in $[28]$ that $\overline{\mathfrak{C}}(J)$ is compact in $\mathcal{E}_{1,1}(M)_{\mathbb{R}}$.

By a variation of the "second separation theorem" [26, p. 65], we get a hyperplane $\mathcal{L}$ in $\mathcal{E}_{1,1}(M)_{\mathbb{R}}$ containing $L$ and disjoint from $\overline{\mathfrak{C}}(J)$. The hyperplane $\mathcal{L}$ determines a functional $\alpha$ vanishing on $L$ and being positive on $\overline{\mathfrak{C}}(J)$. By Lemma 3.3, $\alpha$ is a $J$-compatible symplectic form. Moreover, by construction we have $[\alpha]=e$.

Since $\mathcal{K}_{J}^{c}$ is open in $H_{J}^{1,1}(M)_{\mathbb{R}}$, via the pairing between $H_{J}^{1,1}(M)_{\mathbb{R}}$ and $H_{1,1}^{J}(M)_{\mathbb{R}}, H_{J}^{1,1}(M)_{\mathbb{R}}$ injects into the dual space of $H_{1,1}^{J}(M)_{\mathbb{R}}$. Thus we have 
Corollary 3.1. Suppose $(M, J)$ is an almost complex manifold with $J$ almost Kähler, i.e., $\mathcal{K}_{J}^{c} \neq \emptyset$. Then

$$
\operatorname{dim} H_{J}^{1,1}(M)_{\mathbb{R}} \leq \operatorname{dim} H_{1,1}^{J}(M)_{\mathbb{R}}
$$

The natural questions are whether $H \mathfrak{C}(J)$ is also dual to $\mathcal{K}_{J}^{c}$. If it is true, then when $H \mathfrak{C}(J)$ is open in $H_{1,1}^{J}(M)_{\mathbb{R}}$, we have the equality. This is the case for a Kähler structure.

\subsection{Comparing $\mathcal{K}_{J}^{c}$ and $\mathcal{K}_{J}^{t}$}

We start with

Proposition 3.1. Suppose $(M, J)$ is an almost complex manifold with $J$ almost Kähler, i.e., $\mathcal{K}_{J}^{c} \neq \emptyset$. Then we have

$$
\mathcal{K}_{J}^{t} \cap H_{J}^{1,1}(M)_{\mathbb{R}}=\mathcal{K}_{J}^{c},
$$

and

$$
\mathcal{K}_{J}^{c}+H_{J}^{(2,0),(0,2)}(M)_{\mathbb{R}} \subset \mathcal{K}_{J}^{t}
$$

Proof. Equation (3.4) is a direct consequence of Theorem 3.4 and Theorem 3.2.

For the inclusion (3.5) we can actually explicitly find $J$-tamed symplectic forms in

$$
\mathcal{K}_{J}^{c}+H_{J}^{(2,0),(0,2)}(M)_{\mathbb{R}}
$$

This is discussed in a slightly different context in $[9,25]$. The point is, $\beta \in \Omega_{J}^{(2,0),(0,2)}(M)_{\mathbb{R}}$ vanishes on any complex line, i.e., at any point $x \in M$, $\beta(v, J v)=0$ for $v \in T_{x} M$. Thus for an almost Kähler form $\alpha \in \Omega_{J}^{1,1}(M)_{\mathbb{R}}$, the form $\alpha+t \beta$, restricted to a complex line, is equal to $\alpha$ and hence is positive. If $\beta$ is also closed, then, as already observed in [10], $\alpha+t \beta$ is a $J$-tamed symplectic form for any $t \in \mathbb{R}$.

As mentioned in Remark 2.3, such a $J$ is actually $C^{\infty}$ pure.

We are ready to prove Theorem 1.1 . 
Proof. By Proposition 3.1, it suffices to prove the reverse inclusion

$$
\mathcal{K}_{J}^{c}+H_{J}^{(2,0),(0,2)}(M)_{\mathbb{R}} \supset \mathcal{K}_{J}^{t}
$$

Since $J$ is assumed to be $C^{\infty}$ full, by Theorem 3.4, we have

$$
\text { Int } H \breve{\mathfrak{C}}(J) \subset \mathcal{K}_{J}^{c}+H_{J}^{(2,0),(0,2)}(M)_{\mathbb{R}}
$$

In addition, by Theorem 3.2, Int $H \breve{\mathfrak{C}}(J)=\mathcal{K}_{J}^{t}$. Thus we have the inclusion (3.6).

As a consequence of Theorem 1.1 and Proposition 2.1 we have

Corollary 3.2. Suppose $J$ is a complex structure on $M$. If $\mathcal{K}_{J}^{c} \neq \emptyset$, i.e., $J$ is Kähler, then

$$
\mathcal{K}_{J}^{t}=\mathcal{K}_{J}^{c}+\left(H_{\bar{\partial}}^{2,0}(M) \oplus H_{\bar{\partial}}^{0,2}(M)\right)_{\mathbb{R}}
$$

In particular, if $\mathcal{K}_{J}^{c} \neq \emptyset$, then

$$
\mathcal{K}_{J}^{t} \cap H_{\bar{\partial}}^{1,1}(M)_{\mathbb{R}}=\mathcal{K}_{J}^{c}
$$

\section{Complex structures}

In this section we assume that the almost complex structure $J$ is integrable. For such a $J$ we say that it is Kähler if $\mathcal{K}_{J}^{c}$ is non-empty.

\subsection{Kähler complex structures}

4.1.1. Tamed cones and compatible cones If $J$ is Kähler we have the following type decomposition:

$$
\begin{aligned}
H^{2}(M ; \mathbb{C}) & =H_{\bar{\partial}}^{1,1}(M) \oplus H_{\bar{\partial}}^{2,0}(M) \oplus H_{\bar{\partial}}^{0,2}(M) \\
H^{2}(M ; \mathbb{R}) & =H_{\bar{\partial}}^{1,1}(M)_{\mathbb{R}} \oplus\left(H_{\bar{\partial}}^{2,0}(M) \oplus H_{\bar{\partial}}^{0,2}(M)\right)_{\mathbb{R}}
\end{aligned}
$$

where $H_{\bar{\partial}}^{p, q}$ denotes the $(p, q)$-Dolbeault cohomology group. Notice that, $H \mathfrak{C}(J)$ vanishes on $H_{\bar{\partial}}^{2,0}(M) \oplus H_{\bar{\partial}}^{0,2}(M)$ and thus can be considered to be a subcone of $H_{\bar{\partial}}^{n-1, n-1}(M)_{\mathbb{R}}$. And by the $J$-invariance,

$$
\mathcal{K}_{J}^{c} \subset H_{\bar{\partial}}^{1,1}(M)_{\mathbb{R}}
$$

Moreover, we have the following beautiful description of $\mathcal{K}_{J}^{c}$. 
Theorem 4.1 [5]. Let $J$ be a Kähler complex structure on a real $2 n$ dimensional manifold $M$. Let $T \subset H \mathfrak{C}(J) \subset H_{\bar{\partial}}^{n-1, n-1}(M)_{\mathbb{R}}$ be defined by (2.14). Then the interior of the dual cone of $T$ in $H_{\bar{\partial}}^{1,1}(M)_{\mathbb{R}}$ equals $\mathcal{K}_{J}^{c}$.

An immediate consequence is

Corollary 4.1. Let $J$ be a Kähler complex structure on a real $2 n$ dimensional manifold $M$. Then,

$$
\text { Int } \begin{aligned}
H \mathfrak{C}(J) & =T \\
& =P D\left(\left(\mathcal{K}_{J}^{c}\right)^{n-1}\right)+H C \mathfrak{Y}^{1} \cdot P D\left(\left(\mathcal{K}_{J}^{c}\right)^{n-2}\right)+\cdots+H C \mathfrak{Y}^{n-1},
\end{aligned}
$$

where $H C \mathfrak{Y}^{i} \subset H_{\bar{\partial}}^{i, i}(M)_{\mathbb{R}}$ is generated by classes of $(n-i)$-dimensional irreducible analytic subsets of $M$.

Here is another argument for Corollary 3.2.

Proof. We first show that

$$
\mathcal{K}_{J}^{t} \supset \mathcal{K}_{J}^{c}+\left(H_{\bar{\partial}}^{2,0}(M) \oplus H_{\bar{\partial}}^{0,2}(M)\right)_{\mathbb{R}} .
$$

One proof of (4.3) is to evoke Theorem 3.2. We only need to observe that $\left(H_{\bar{\partial}}^{2,0}(M) \oplus H_{\bar{\partial}}^{0,2}(M)\right)_{\mathbb{R}}$ vanishes on $H \mathfrak{C}(J)$ and $\mathcal{K}_{J}^{c}$ is positive on $H \mathfrak{C}(J)$. Thus $\mathcal{K}_{J}^{c}+\left(H_{\bar{\partial}}^{2,0}(M) \oplus H_{\bar{\partial}}^{0,2}(M)\right)_{\mathbb{R}}$ is in the interior of the dual of $H \mathfrak{C}(J)$.

To prove the other direction, we apply Theorem 4.1 to obtain

$$
\text { Int } H \breve{\mathfrak{C}}(J)=\operatorname{Int} \breve{T} \subset \mathcal{K}_{J}^{c}+\left(H_{\bar{\partial}}^{2,0}(M) \oplus H_{\bar{\partial}}^{0,2}(M)\right)_{\mathbb{R}} .
$$

To state the next result let us introduce a definition.

Definition 4.1. A degree 2 real cohomology class $V$ of a complex manifold $M$ is said to be numerically positive on analytic cycles, or numerically positive for short, if $V^{p}$ pairs positively with the homology class of $Y$ for any irreducible analytic set $Y$ in $M$ with $\operatorname{dim}_{\mathbb{C}} Y=p$. Let $\mathcal{N P}$ denote the set of numerically positive classes.

We have the following characterization of the $J$-tamed cone in terms of numerically positive classes.

Theorem 4.2. Let $(M, J)$ be a compact Kähler manifold. Then the J-tamed cone $\mathcal{K}_{J}^{t}$ is one of the connected components of $\mathcal{N} \mathcal{P}$. 
Proof. Since the space of $J$-tamed symplectic forms is a convex set the cone $\mathcal{K}_{J}^{t}$ is connected. Thus it suffices to show the following two inclusions: one component of $\mathcal{N} \mathcal{P} \subset \mathcal{K}_{J}^{t}$ and $\mathcal{K}_{J}^{t} \subset \mathcal{N} \mathcal{P}$.

Let $\mathcal{N} \mathcal{P}^{1,1}$ be the $(1,1)$ part of $\mathcal{N} \mathcal{P}$. Then by the main result of [5] (cf. Theorem 4.5) one component of $\mathcal{N P}^{1,1}$ is contained in $\mathcal{K}_{J}^{c}$. Denote this component by $\widetilde{\mathcal{N} \mathcal{P}^{1,1}}$. Then by (3.7) we have

$$
\widetilde{\mathcal{N} \mathcal{P}^{1,1}}+\left(H_{\bar{\partial}}^{2,0}(M) \oplus H_{\bar{\partial}}^{0,2}(M)\right)_{\mathbb{R}} \subset \mathcal{K}_{J}^{c}+\left(H_{\bar{\partial}}^{2,0}(M) \oplus H_{\bar{\partial}}^{0,2}(M)\right)_{\mathbb{R}}=\mathcal{K}_{J}^{t} .
$$

On the other hand, the component of $\mathcal{N P}$ which contains $\widetilde{\mathcal{N} \mathcal{P}^{1,1}}$ definitely belongs to

$$
\widetilde{\mathcal{N P}^{1,1}}+\left(H_{\bar{\partial}}^{2,0}(M) \oplus H_{\bar{\partial}}^{0,2}(M)\right)_{\mathbb{R}} .
$$

Thus we have proved the first inclusion.

To prove $\mathcal{K}_{J}^{t} \subset \mathcal{N} \mathcal{P}$, write any $V \in \mathcal{K}_{J}^{t}$ as $U+W$, where $U$ is the $(1,1)$ part. By (3.7), $U=[\alpha]$ for a Kähler form $\alpha$ and $W=[\beta]$ for a real closed form in $\Omega_{J}^{2,0}(M) \oplus \Omega_{J}^{0,2}(M)$. Then $\alpha+\beta$ is a tamed symplectic form when restricted to the smooth part of any irreducible analytic set $Y$. It follows that $V$ is numerically positive, i.e., $\mathcal{K}_{J}^{t} \subset \mathcal{N} \mathcal{P}$.

4.1.2. Relating to some classical theorems Corollary 3.2 and Theorem 4.2 are parallel to some classical theorems in Kähler geometry and algebraic geometry.

Let us recall some basic concepts in algebraic geometry. For a complex manifold $(M, J)$ a very ample line bundle $L$ is a holomorphic line bundle on $M$ with enough holomorphic sections to set up an embedding of $M$ into a projective space. An ample line bundle $L$ is one whose certain tensor power becomes very ample. Taking the first Chern class of every ample line bundle we obtain a cone $\mathcal{A}$, called the ample cone. Clearly $\mathcal{A}$ lies in the integral $(1,1)$ cohomology group, i.e.,

$$
\mathcal{A} \subset H^{1,1}(M ; \mathbb{Z}):=H^{2}(M ; \mathbb{Z}) \cap H^{1,1}(M ; \mathbb{R}) .
$$

In this subsection we will simply write $H^{1,1}(M ; \mathbb{R})$ for $H_{\bar{\partial}}^{1,1}(M)_{\mathbb{R}}$.

Recall we denote the Kähler cone by $\mathcal{K}_{J}^{c} \subset H^{1,1}(M ; \mathbb{R})$. The classical Kodaira embedding theorem can then be stated in the following way as a comparison of the triples

(projective, $\left.H^{1,1}(M ; \mathbb{Z}), \mathcal{A}\right)$ and $\quad\left(\right.$ Kähler, $\left.H^{1,1}(M ; \mathbb{R}), \mathcal{K}_{J}^{c}\right)$. 
Theorem 4.3. Let $(M, J)$ be a Kähler manifold. If $\mathcal{A} \neq \emptyset$, then

$$
\mathcal{A}=\mathcal{K}_{J}^{c} \cap H^{1,1}(M ; \mathbb{Z})
$$

With this interpretation of the Kodaira embedding theorem our Proposition 3.1 can then be viewed as a (Kähler, $J$-symplectic) analogue comparing the triples

$\left(\right.$ Kähler, $\left.H^{1,1}(M ; \mathbb{R}), \mathcal{K}_{J}^{c}\right) \quad$ and $\quad\left(J-\right.$ symplectic, $\left.H^{2}(M ; \mathbb{R}), \mathcal{K}_{J}^{t}\right)$.

Here, a $J$-symplectic manifold means a manifold with a symplectic form tamed by a complex structure $J$. To see that let $(M, J)$ be a complex manifold with non-empty tamed cone $\mathcal{K}_{J}^{t}$. Then Equation (4.5) is exactly the analogue of Equation (3.8).

In addition, our Theorem 4.2 is a Nakai-Moishezon type theorem in the $J$-symplectic world. Recall that the original Nakai-Moishezon characterizes the ample cone in terms of numerically positive classes in Definition 4.1.

Theorem 4.4. The ample cone $\mathcal{A}$ of a projective manifold $(M, J)$ is given by

$$
\mathcal{A}=\mathcal{N} \mathcal{P} \cap H^{1,1}(M ; \mathbb{Z})
$$

Recall also the recent remarkable extension of Demailly-Paun in [5] (first established in $[3,17]$ in the case of $n=2$ ).

Theorem 4.5. The Kähler cone of a Kähler manifold $(M, J)$ is one of the connected components of

$$
\mathcal{N P} \cap H^{1,1}(M ; \mathbb{R})
$$

Theorem 4.2 can be trivially restated as that the tamed cone of a Kähler manifold $(M, J)$ is one of the connected components of

$$
\mathcal{N P} \cap H^{2}(M ; \mathbb{R})
$$

The parallel between (4.6), (4.7) and (4.8) is clear. Especially, in dimension 4 , by virtue of the second statement of Theorem 1.2 , we can replace the first sentence in Theorem 4.5, "a compact Kähler manifold $(M, J)$ ", by "a compact $J$-symplectic surface $(M, J)$ ". Thus, at least in dimension 4, 
replacing the triple

$$
\left(J \text { - symplectic, } H^{2}(M ; \mathbb{R}), \mathcal{K}_{J}^{t}\right)
$$

by the triple

$$
\text { (projective, } \left.H^{1,1}(M ; \mathbb{Z}), \mathcal{A}\right)
$$

specializes to the classical Nakai-Moishezon theorem.

\subsection{Complex surfaces}

In this subsection we specialize to complex dimension 2 .

4.2.1. Tamed cones and compatible cones We start with the proof of Theorem 1.2.

Proof. If $\mathcal{K}_{J}^{t}(M)$ is empty, then clearly $\mathcal{K}_{J}^{c}(M)$ is empty as well.

Suppose $\mathcal{K}_{J}^{c}(M)$ is empty. Then it follows from $[3,24,28,29]$ that $b_{1}(M)$ is odd. If $b^{+}(M)=0$ then $M$ has no symplectic structures, hence $\mathcal{K}_{J}^{t}(M)$ is empty. The remaining case is $b_{1}$ odd and $b^{+} \geq 1$. In this case, by the Kodaira classification of surfaces (see [4]), $(M, J)$ is elliptic. By [16], for an elliptic surface $(M, J)$ with $b_{1}$ odd, the torus fibers bound, thus there cannot be tamed symplectic forms.

Example 4.1. The Kodaira-Thurston manifold is an example with $b_{1}$ odd. Thus $\mathcal{K}_{J}^{t}$ is empty for every integrable $J$. But it does admit symplectic structures hence $\mathcal{K}_{J}^{t}$ is not empty for some non-integrable $J$.

It follows from Theorem 3.2 and Corollary 4.1 that, for a complex surface $(M, J), H \mathfrak{C}(J)$ contains the 0 element if $J$ is non-Kähler, and is equal to $P D\left(\mathcal{K}_{J}^{c}\right)+H C \mathfrak{Y}$ if $J$ is Kähler.

Remark 4.1. In this case a slightly different description of $\mathcal{K}_{J}^{c}$ was first proved in $[3,17]$ by Buchdahl and Lamari independently: Let $(M, J)$ be a complex surface. If $\mathcal{K}_{J}^{c}$ is non-empty, then $\mathcal{K}_{J}^{c}$ is a connected component of

$$
\left\{U \in H_{\bar{\partial}}^{1,1}(M)_{\mathbb{R}} \mid U^{2}>0 \text { and } U \text { is positive on } H C \mathfrak{Y}\right\},
$$

where $H C \mathfrak{Y}$ is the convex cone in $H^{2}(M ; \mathbb{R})$ generated by classes of irreducible holomorphic curves. 
Let $C^{-} \mathfrak{Y}$ denote the subcone generated by irreducible curves of negative square. It follows from the Hodge index theorem that the Kähler cone $K_{J}^{c}$ is given by a connected component of

$$
\left\{U \in H_{\bar{\partial}}^{1,1}(M)_{\mathbb{R}} \mid U^{2}>0 \text { and } U \text { is positive on } H C^{-} \mathfrak{Y}\right\} \text {. }
$$

In particular, if there are no irreducible curves of negative square, the Kähler cone coincides with one connected component of the $(1,1)$ positive cone. Such examples include generic complex structures on $K 3$ or $T^{4}$.

In higher dimensions we speculate that, for an integrable $J$ it is possible that $K_{J}^{c}$ is empty while $K_{J}^{t}$ is non-empty. Such examples could come from the total space of a holomorphic bundle over a Kähler manifold admitting a class whose restriction is in the tamed cone of each fiber. In such a situation, on the one hand, there is the construction of tamed symplectic forms in [15], and on the other hand, there are new subtle obstructions being Kähler in [33]. Here is a potential example inspired by [33].

Example 4.2. Let $T^{10}$ be the real torus of dimension 10, and let $K$ be any simply connected compact Kähler manifold satisfying the conditions that rank $H^{2}(K ; \mathbb{Q})=11$ and that the cohomology of $K$ is generated in degree 2. By Künneth decomposition and Poincaré duality we have an inclusion $\operatorname{Hom}\left(H^{2}\left(T^{10} ; \mathbb{Q}\right), H^{2}(K ; \mathbb{Q})\right) \subset H^{10}\left(T^{10} \times K ; \mathbb{Q}\right)$. Consider a generic surjective $\operatorname{map} \mu: H^{2}\left(T^{10} ; \mathbb{Q}\right) \rightarrow H^{2}(K ; \mathbb{Q})$ and let $\lambda \in H^{10}\left(T^{10} \times K ; \mathbb{Q}\right)$ be the image of $\mu$ under the inclusion.

Suppose there is a holomorphic bundle $E$ over $Y:=T^{10} \times K$ such that

$$
c_{1}(E)=0, \quad \text { and } \quad c_{5}(E)=m \lambda
$$

for some non-zero integer $m$. Consider the complex manifold $\mathbb{P}(E)$. By Theorem 3.5 in [33], the complex manifold $\mathbb{P}(E)$ is not Kähler.

On the other hand, since the first Chern class of the tautological line bundle of $\mathbb{P}(E)$ restrict to a symplectic class of each fiber, the complex manifold $\mathbb{P}(E)$ admits a tamed symplectic structure by [15].

\subsubsection{Integrable tamed cone versus integrable compatible cone} In this subsection let us now change our perspective slightly. Rather than fixing a pair $(M, J)$ we fix a manifold $M$ and consider all possible complex structures $J$ on $M$. 
Definition 4.2. Let $\mathcal{K}_{\text {Int }}^{t}(M)$ be the union of $\mathcal{K}_{J}^{t}(M)$ over all integrable $J$ on $M$, called the integrable tamed cone of $M$. Similarly define the integrable compatible cone $\mathcal{K}_{\text {Int }}^{c}(M)$.

$\mathcal{K}_{\text {Int }}^{c}(M)$ is called the cohomology Kähler cone in $[9,25]$. In general, the integrable tamed cone is not equal to the integral compatible cone. For example, for a ball quotient $\left(M, J_{0}\right)$, the complex structure $J_{0}$ is the unique complex structure, thus by Equation (3.7)

$$
\mathcal{K}_{\text {Int }}^{c}(M)=\mathcal{K}_{J_{0}}^{c}(M) \subsetneq \mathcal{K}_{J_{0}}^{t}(M)=\mathcal{K}_{\text {Int }}^{t}(M),
$$

if $H_{\bar{\partial}}^{2,0}(M) \neq 0$.

We can also fix a degree 2 class $e$ in $H^{2}(M ; \mathbb{Z})$ and consider the subcone $\mathcal{K}_{\text {Int }}^{t}(M, e)$ of $\mathcal{K}_{\text {Int }}^{t}(M)$, called the $e$-integrable tamed cone of $M$. It is the union of $\mathcal{K}_{J}^{t}(M)$ over all integrable $J$ on $M$ with $e$ being the first Chern class. Similarly we have the subcone $\mathcal{K}_{\text {Int }}^{c}(M, e)$ of $\mathcal{K}_{\text {Int }}^{c}(M)$, the $e$-integrable compatible cone of $M$.

In the case that $e$ is realized by $-\omega$ for some Kähler form $\omega$ on $M$, by the Hodge-Riemann bilinear relations, it can be shown that

$$
\mathcal{K}_{\text {Int }}^{c}(M, e) \subsetneq \mathcal{K}_{\text {Int }}^{t}(M, e),
$$

in certain situations. More precisely, let $J$ be a complex structure for which $-e=[\omega]$ where $\omega$ is a Kähler form for $J$. Suppose $\beta$ is a non-zero real and closed form in $\Omega_{J}^{2,0}(M) \oplus \Omega_{J}^{0,2}(M)$. Results in $[9,10,25]$ can be interpreted as saying that $-e+t[\beta]$ is not in $\mathcal{K}_{\text {Int }}^{c}(M, e)$ if

- $n$ is even, $\beta^{\frac{n}{2}}$ is not identically zero, and $|t|$ is sufficiently large, or

- $n=2, t \neq 0$.

We further observe that the same is true for $n$ odd and $|t| \neq 0$ sufficiently small. On the other hand, as already used in the proof of Proposition 3.1, $\omega+t \beta$ is tamed by $J$ for any $t$. Thus in these cases we have the strict inclusion (4.9).

Coming back to the cones $\mathcal{K}_{\text {Int }}^{c}(M)$ and $\mathcal{K}_{\text {Int }}^{t}(M)$, we have the following conjecture in the case of $n=2$.

Conjecture 4.1. For any $M$ underlying a complex surface of general type with $p_{g} \geq 1$, we have

$$
\mathcal{K}_{\text {Int }}^{c}(M) \subsetneq \mathcal{K}_{\text {Int }}^{t}(M)
$$


and for all other 4-dimensional manifolds, we have the the equality

$$
\mathcal{K}_{\text {Int }}^{c}(M)=\mathcal{K}_{\text {Int }}^{t}(M) .
$$

This conjecture is plausible. In the case $n=2$, for a smooth manifold $M$ underlying a minimal surface of general type, by the Donaldson theory or the Seiberg-Witten theory (c.f. $[2,13,14])$, the set $\left\{c_{1}(M, J),-c_{1}(M, J)\right\}$ is the same for any $J$. Consequently, we have the strict inclusion (4.10) if $b^{+}(M) \geq 3$ and there is a complex structure on $M$ together with a Kähler form on $(M, J)$ representing its canonical class.

Secondly, an immediate consequence of Theorem 1.2 is that if $M$ has dimension 4 then $\mathcal{K}_{\text {Int }}^{t}(M)$ is empty if and only if $\mathcal{K}_{\text {Int }}^{c}(M)$ is empty.

Thirdly, if $p_{g}=0$ then $H^{2}(M ; \mathbb{R})=H_{\bar{\partial}}^{1,1}(M)_{\mathbb{R}}$ and hence $\mathcal{K}_{J}^{t}=\mathcal{K}_{J}^{c}$ by (3.7). Therefore we have (4.11) in this case.

Finally, when $p_{g}>0$, the equality (4.11) still holds when the Kodaira dimension is zero (see [20]). We speculate that it is also true when the Kodaira dimension is one, in particular, for the Elliptic surfaces $E(n)$.

\section{Acknowledgments}

The authors wish to thank S-T. Yau for stimulating questions. We also appreciate F. Zheng, S. Morgan, V. Tosatti, B. Weinkove for discussions, S. Donaldson, M. Furuta, R. Hind, K. Ono for their interest, A. Fino, A. Tomassini for sending to us their preprint [12]. We are grateful to T. Drăghici for his interest and pointing out reference [10]. Finally, we would like to thank the referee for careful reading and very useful comments. The authors are partially supported by NSF and the McKnight foundation.

\section{References}

[1] V. Apostolov and T. Drăghici, The curvature and the integrability of almost-Kähler manifolds: a survey, Symplectic and contact topology: interactions and perspectives (Toronto, ON/Montreal, QC, 2001), 25-53, Fields Inst. Commun., 35, American Mathematic Soceity, Providence, RI, 2003.

[2] R. Brussee, The canonical class and the $C^{\infty}$ properties of Kähler surfaces, New York J. Math. 2 (1996), 103-146.

[3] N. Buchdahl, On compact Kähler surfaces, Ann. Inst. Fourier (Grenoble) 49(1) (1999), vii, xi, 287-302. 
[4] W. Barth, K. Hulek, C. Peters and A. Van de Ven, Compact complex surfaces, Ergebnisse der Mathematik und ihrer Grenzgebiete. 3. Folge. A Series of Modern Surveys in Mathematics [Results in Mathematics and Related Areas. 3rd Series. A Series of Modern Surveys in Mathematics], 4. Springer-Verlag, Berlin, 2004.

[5] J. Demailly, M. Paun, Numerical characterization of the Kähler cone of a compact Kähler manifold, Ann. of Math. (2) 159(3), (2004), 12471274.

[6] G. de Rham, Differentiable manifolds. Forms, currents, harmonic forms, Grundlehren der Mathematischen Wissenschaften [Fundamental Principles of Mathematical Sciences], 266, Springer-Verlag, Berlin, 1984, x+167pp.

[7] S.K. Donaldson, Two-forms on four-manifolds and elliptic equations, Inspired by S. S. Chern, 153-172, Nankai Tracts Math., 11, World Sci. Publ., Hackensack, NJ, 2006.

[8] J.G. Dorfmeister and T.-J. Li, The relative symplectic cone and $T^{2}$ fibrations, arXiv:0805.2957, to appear in J. Symplectic Geom.

[9] T. Drăghici, The Kähler cone versus the symplectic cone, Bull. Math. Soc. Sci. Math. Roumanie (N.S.) 42(90)(1) (1999), 41-49.

[10] T. Drăghici, Symplectic obstructions to the existence of $\omega$-compatible Einstein metrics, Differential Geom. Appl. 22(2) (2005), 147-158.

[11] T. Drăghici, T.-J. Li and W. Zhang, Symplectic forms and cohomology decomposition of almost complex 4-manifolds, arXiv:0812.3680, to appear in IMRN, doi:10.1093/imrn/rnp113

[12] A. Fino and A. Tomassini, On some cohomological properties of almost complex manifolds, arXiv:0811.4765, to appear in J. Geom. Anal.

[13] R. Friedman and J. Morgan, Algebraic surfaces and Seiberg-Witten invariants, J. Algebraic Geom. 6 (1997), 445-479.

[14] R. Friedman and Z. Qin, On complex surfaces diffeomorphic to rational surfaces, Invent. Math. 120 (1995), 81-117.

[15] R. Gompf, Toward a topological characterization of symplectic manifolds, J. Symplectic Geom. 2(2) (2004) 177-206.

[16] R. Harvey and H. Lawson, An intrinsic characterization of Kähler manifolds, Invent. Math. 74(2) (1983), 169-198. 
[17] A. Lamari, Courants Kählériens et surfaces compactes, Ann. Inst. Fourier (Grenoble) 49(1) (1999), vii, x, 263-285.

[18] J. Lee, Family Gromov-Witten invariants for Kähler surfaces, Duke Math. J. 123(1) (2004), 209-233.

[19] M. Lejmi, Strictly nearly Kähler 6-manifolds are not compatible with symplectic forms, C. R. Math. Acad. Sci. Paris 343(11-12) (2006), 759-762.

[20] T.-J. Li, The space of symplectic structures on closed 4-manifolds, Third International Congress of Chinese Mathematicians. Part 1, 2, 259-277, AMS/IP Stud. Adv. Math., 42, part.1, 2, Amer. Math. Soc., Providence, RI, 2008.

[21] T.-J. Li and A.-K. Liu, Uniqueness of symplectic canonical class, surface cone and symplectic cone of 4-manifolds with $B^{+}=1$, J. Differential Geom. 58(2) (2001), 331-370.

[22] T.-J. Li and M. Usher, Symplectic forms and surfaces of negative square, J. Symplectic Geom. 4(1) (2006), 71-91.

[23] D. McDuff, Notes on ruled symplectic 4-manifolds, Trans. Amer. Math. Soc. 345(2) (1994), 623-639.

[24] Y. Miyaoka, Kähler metrics on elliptic surfaces, Proc. Japan Acad. 50 (1974), 533-536.

[25] D. Perrone, A characterization of cohomological Einstein Kaehler manifolds and applications, Geom. Dedicata 22 (1987), 255-260.

[26] H. Schaefer, Topological vector spaces, Springer-Verlag, Berlin, 1971.

[27] Y. T. Siu, Every K3 surface is Kähler, Invent. Math. 73(1) (1983), 139-150.

[28] D. Sullivan, Cycles for the dynamical study of foliated manifolds and complex manifolds, Invent. Math. 36 (1976), 225-255.

[29] Todorov, Applications of the Kähler-Einstein-Calabi-Yau metric to moduli of K3-surfaces, Invent. Math. 81 (1980), 251-266.

[30] V. Tosatti, B. Weinkove and S.T. Yau, Taming symplectic forms and the Calabi-Yau equation, Proc. Lond. Math. Soc. (3) 97(2) (2008), 401-424. 
[31] A. Tomassini, Some examples of non calibrable almost complex structures, Forum Math. 14 (2002), 869-876.

[32] B. Weinkove, The Calabi-Yau equations on almost Kahler manifolds, J. Differential Geom., 76(2) (2007), 317-349.

[33] C. Voisin, Hodge structures on cohomology algebras and geometry, Math. Ann. 341(1) (2008), 39-69.

School of Mathematics

UnIVERSITY OF MinnESOTA

MinNEAPOLIS

MN 55455

USA

E-mail address: tjli@math.umn.edu; zhang393@math.umn.edu

Received March 21, 2009 
